\title{
Signal Velocity in Oscillator Arrays
}

\author{
Carlos E. Cantos * $^{*}$ J. J. P. Veerman ${ }^{\dagger}$ \\ David K. Hammond $\ddagger$
}

February 26, 2018

\begin{abstract}
We investigate a system of coupled oscillators on the circle, which arises from a simple model for behavior of large numbers of autonomous vehicles. The model considers asymmetric, linear, decentralized dynamics, where the acceleration of each vehicle depends on the relative positions and velocities between itself and a set of local neighbors. We first derive necessary and sufficient conditions for asymptotic stability, then derive expressions for the phase velocity of propagation of disturbances in velocity through this system. We show that the high frequencies exhibit damping, which implies existence of well-defined signal velocities $c_{+}>0$ and $c_{-}<0$ such that low frequency disturbances travel through the flock as $f\left(x-c_{+} t\right)$ in the direction of increasing agent numbers and $f\left(x-c_{-} t\right)$ in the other.
\end{abstract}

\section{Introduction}

This paper is part of a larger program to develop mathematical methods to quantitatively study performance of models for flocking. The main underlying motivation for the current work is to inform development of methods for programming driverless cars to enable coherent motion at high speed, even under dense traffic conditions. This is obviously an important problem, not only because it can lead to enormous cost savings to have smooth and dense traffic on our busier highways, but also because failures may cost lives.

We study models that assume that each car is programmed identically and that can observe relative velocities and positions of nearby cars. In this work we take nearby to mean only the car in front and behind. However the methods we

\footnotetext{
* Maseeh Department of Mathematics \& Statistics, Portland State University, Portland, OR. Email : ccantos@pdx.edu

${ }^{\dagger}$ Maseeh Department of Mathematics \& Statistics, Portland State University, Portland, OR, and CCQCN, Dept of Physics, University of Crete, Heraklion, Greece. Email : veerman@pdx.edu

${ }^{\ddagger}$ Applied Mathematics Department, Oregon Institute of Technology, Wilsonville, OR. Email : david.hammond@oit.edu
} 
develop will be applicable to larger interactions (and these will be explored in future work). We will assume that the system is linearized. Various examples and analyses of nonlinear systems exist. But the emphasis here is on linear systems where we can allow for many parameters (to take the neighbors into account) and still perform a meaningful analysis.

There are two main aspects in our analysis. The first is the asymptotic stability. This can be analyzed via the eigenvalues of the matrix associated with the first order differential equation. Section 3 is devoted to establishing necessary and sufficient conditions for a class of systems to be asymptotically stable. Even though this is a fairly straightforward calculation, we have not found it in this generality in the literature.

The second, more delicate aspect of the problem is related to the fact that we may have arbitrarily many cars following each other, hundreds or even thousands. In this situation, even if all our systems are known to be asymptotically stable, transients may still grow exponentially in the number of cars. The spectrum of the linear operator does not help us to recognize this problem ([16]). A dramatic example of this can be found in [18, where eigenvalues have real part bounded from above by a negative number and yet transients grow exponentially in $N$. This kind of exponential growth underscores the need for different (non-spectral) methods to analyze these systems. The main result of our paper represents one such alternative approach. We establish that for the parameter values of interest (e.g. asymptotically stable systems), solutions are well approximated by travelling wave signals with two distinct signal velocities, one positive (in direction of increasing agent number) and one negative.

Ever since the inception ([6], [9]) of the subject, systems with periodic boundary conditions have been popular ([7, [8, and [1]) because they tend to be easier to study. However the precise connection between these systems and more realistic systems with non-trivial boundary conditions has always been somewhat unclear. Our current program differs from earlier work in two crucial ways. The first is that we make precise what the impact of our analysis is for the (more realistic) systems on the line : namely in this paper we derive an expression for the velocity with which disturbances propagate in systems with periodic boundary, and in 4] we numerically verify that this holds on the line as well. The second is that we consider all possible nearest neighbor interactions: we do not impose symmetries. This turns out to be of the utmost importance: when we apply these ideas in [4] it turns out that the systems with the best performance are asymmetric. Asymmetric systems (though not the same as ours) have also been considered by 2 and with similar results. However their methods are perturbative, and spectral based. In [15] and 11] asymmetric interactions are also studied, and it was shown that in certain cases they may lead to exponential growth (in $N$ ) in the perturbation. In the later of these, the model is qualitatively different because absolute velocity feedback is assumed (their method is also perturbative and not global). Signal velocities were employed in earlier calculations namely [19] and [12. These calculations have in common that they were done for car-following models. We are interested in a more general framework, namely where automated pilots may pay attention also to their neighbor 
behind them or indeed other cars further afield.

Our model is strictly decentralized. There are two reasons to do that. First, in high speed, high/density traffic, small differences in measured absolute velocity may render that measurement useless, if not dangerous, for the feedback. Secondly, the desired velocity, even on the highway, may not be constant. It will depend on weather, time of day, condition of the road, and so on. For these reasons we limit ourselves to strictly decentralized models that only use information relative to the observers in the cars (see [17] and [18]). Many authors study models featuring a term proportional to velocity minus desired velocity (see e.g. [1, 2], [6], 7], 8], 9], and [11]).

\section{Flocking Model}

We consider a model of a decentralized flock of $N$ moving agents (e.g. cars), where each agent's acceleration depends linearly on on the differences between its own relative position and velocity, and those of some subset of neighbors. Letting $x_{k}$ be the position of the $k^{t h}$ agent, and $h_{k}$ its desired distance within the flock (typically $k$ times a fixed spacing $\delta$ ), the general linear decentralized flock satisfies

$$
\ddot{x}_{k}=\sum_{j \in \mathcal{N}_{k}} p_{j, k}\left(\left(x_{k}-h_{k}\right)-\left(x_{j}-h_{j}\right)\right)+v_{j, k}\left(\dot{x}_{k}-\dot{x}_{j}\right)
$$

where $\mathcal{N}_{k}$ is the set of neighbors for agent $k$, and $p_{j, k}$ and $v_{j, k}$ are the coefficients for how the difference of positions and velocities respectively between agent $k$ and $j$ affect the acceleration of agent $k$. The above model is more general than that considered in this work, we restrict ourselves to a leaderless decentralized flock with identical agents and periodic boundary. These restrictions imply $p_{k, j}$ and $v_{k, j}$ depend only on $j-k \bmod N$, and that the neighborhood sets $\mathcal{N}_{k}$ be shift invariant, e.g. $\mathcal{N}_{j+k}=\left\{(j+i) \bmod N: i \in \mathcal{N}_{k}\right\}$. We will also restrict ourselves to nearest neighbor systems. To further simplify the resulting equations, we introduce the change of variables $z_{k} \equiv x_{k}-h_{k}$ (see [17] for more details). We also introduce constants $g_{x}$ and $g_{v}$, define $\rho_{x, j}=\frac{1}{g_{x}} p_{j, 0}$ for $j \neq 0$ and $\rho_{x, 0}=\frac{1}{g_{x}} \sum_{j \neq 0} p_{j, 0}$ where all indices are treated $\bmod N$, and define $\rho_{v, j}$ similarly. It will be convenient to allow negative indices for $\rho_{x, j}$ by setting $\rho_{x, j+N}=\rho_{x, j}$, similarly for $\rho_{v, j}$. In this notation, the flock equations become the following :

Definition 2.1. The system $S_{N}^{*}$ is given by the equation

$$
\ddot{z}_{k}=g_{x} \sum_{j \in \mathcal{N}} \rho_{x, j} z_{k+j}+g_{v} \sum_{j \in \mathcal{N}} \rho_{v, j} \dot{z}_{k+j} \equiv g_{x} \sum_{j=1}^{N} L_{x, k, j} z_{j}+g_{v} \sum_{j=1}^{N} L_{v, k, j} \dot{z}_{j}
$$

where $\mathcal{N}=\{-1,0,1\}$. The $N \times N$ matrices $L_{x}$ and $L_{v}$ defined implicitly above are circulant matrices, as $L_{x, k, j}$ and $L_{v, k, j}$ depend only on $j-k \bmod N$. They 
also have row sums equal to 0 , as the decentralized condition has implied that

$$
\sum_{j \in \mathcal{N}} \rho_{x, j}=\sum_{j \in \mathcal{N}} \rho_{v, j}=0 .
$$

We will accordingly refer to $L_{x}$ and $L_{v}$ as Laplacian matrices.

Remark : It is well known that circulant matrices have orthogonal eigenbases, and are diagonalized by the discrete Fourier transform (see [10]). This is the reason periodic boundary conditions are so convenient.

It will be useful to write the equations of $S_{N}^{*}$ as a first order system:

$$
\frac{d}{d t}\left(\begin{array}{l}
z \\
\dot{z}
\end{array}\right)=M_{N}\left(\begin{array}{l}
z \\
\dot{z}
\end{array}\right) \equiv\left(\begin{array}{cc}
0 & I \\
g_{x} L_{x} & g_{v} L_{v}
\end{array}\right)\left(\begin{array}{l}
z \\
\dot{z}
\end{array}\right)
$$

This system has a 2-dimensional family of coherent solutions, namely:

$$
\forall i \quad z_{i}(t)=v_{0} t+x_{0}
$$

where $v_{0}$ and $x_{0}$ are arbitrary elements of $\mathbb{R}$. These correspond to the generalized eigenspace of $M_{N}$ for the eigenvalue 0 . It is easy to see that all solutions converge to one of these coherent solutions if and only if all other eigenvalues of $M_{N}$ have negative real part. With a slight abuse of notation we will call this case asymptotically stable (see [13] for precise definitions):

Definition 2.2. The system in Equation 3 is called asymptotically stable if it has a single eigenvalue equal to 0 with algebraic multiplicity 2 , and all other eigenvalues have strictly negative real parts.

The discrete Fourier transform will play a fundamental role in our analysis. We define $\lambda_{x, m}$ and $\lambda_{x, m}$ as follows: denote $\theta \equiv \frac{2 \pi}{N}$ and set

$$
\lambda_{x, m} \equiv g_{x} \sum_{j \in \mathcal{N}} \rho_{x, j} e^{i j m \theta} \quad \text { and } \quad \lambda_{v, m} \equiv g_{v} \sum_{j \in \mathcal{N}} \rho_{v, j} e^{i j m \theta}
$$

Denote the vector $w_{m}$ by:

$$
w_{m} \equiv \frac{1}{\sqrt{N}}\left(1, e^{i m \theta}, e^{2 i m \theta}, \cdots e^{(N-1) i m \theta}\right)^{T}
$$

We furthermore define the moments of $g_{x} \rho_{x}$ and $g_{v} \rho_{v}$ :

$$
I_{x \ell} \equiv g_{x} \sum_{j \in \mathcal{N}} \rho_{x, j} j^{\ell} \quad \text { and } \quad I_{v \ell} \equiv g_{v} \sum_{j \in \mathcal{N}} \rho_{v, j} j^{\ell}
$$

and observe that $\lambda_{x, m}$ can be expanded as

$$
\lambda_{x, m}=i m \theta I_{x, 1}-\frac{m^{2} \theta^{2}}{2} I_{x, 2}-i \frac{m^{3} \theta^{3}}{3 !} I_{x, 3}+\frac{m^{4} \theta^{4}}{4 !} I_{x, 4}+i \frac{m^{5} \theta^{5}}{5 !} I_{x, 5} \ldots
$$

An analogous expansion for $\lambda_{v, m}$ can also be given. 


\section{Asymptotic Stability}

In this section we state and prove necessary and sufficient conditions for nearest neighbor systems to be asymptotically stable.

Proposition 3.1. Let $L_{x}$ and $L_{v}$ be the Laplacians defined in Definition 2.1 The eigenvalues of $g_{x} L_{x}$ are $\lambda_{x, m}$ with associated eigenvector $w_{m}$ (where $m \in$ $\{0, \cdots, N-1\})$. Similarly, $\lambda_{v, m}$ and $w_{m}$ form eigenpairs for $g_{v} L_{v}$.

Proof. This follows immediately from the previous remark as $L_{x}$ and $L_{v}$ are circulant matrices.

Remark: Even though $L_{x}$ and $L_{v}$ have bases of orthogonal eigenvectors, $M_{N}$ does not. Instead, the eigenvectors of the $2 N \times 2 N$ matrix $M_{N}$ lie within $N$ twodimensional subspaces which are orthogonal to each other. Each of these may be spanned by two not necessarily orthogonal eigenvectors, or by an eigenvector and a (Jordan) generalized eigenvector. This is made precise below:

Proposition 3.2. The eigenvalues $\nu_{m \pm}(m \in\{0, \cdots N-1\})$ of $M$ are given by the solutions of

$$
\nu^{2}-\lambda_{v, m} \nu-\lambda_{x, m}=0 \quad \Rightarrow \quad \nu_{m \pm}=\frac{\lambda_{v, m}}{2} \pm \sqrt{\frac{\lambda_{v, m}^{2}}{4}+\lambda_{x, m}}
$$

with associated eigenvectors given by $\left(\begin{array}{c}w_{m} \\ \nu_{m \pm} w_{m}\end{array}\right)$.

Proof. Let $\nu$ be an eigenvalue of $M_{N}$, with eigenvector written as $\left(\begin{array}{l}q \\ u\end{array}\right)$. Then

$$
\left(\begin{array}{cc}
0 & I \\
g_{x} L_{x} & g_{v} L_{v}
\end{array}\right)\left(\begin{array}{l}
q \\
u
\end{array}\right)=\nu\left(\begin{array}{l}
q \\
u
\end{array}\right)
$$

which implies first that $u=\nu q$ and then that $\left(g_{x} L_{x}+g_{v} L_{v} \nu\right) q=\nu^{2} q$. The latter shows that $\nu^{2}$ is an eigenvalue of the circulant matrix $g_{x} L_{x}+\nu g_{v} L_{v}$, which from Proposition 3.1 has eigenvalues given by $\lambda_{x, m}+\nu \lambda_{v, m}$, for $0 \leq m \leq N-1$. This implies $\nu$ satisfies $\nu^{2}=\lambda_{x, m}+\nu \lambda_{v, m}$ for some $m$. Finally, letting $\nu_{m \pm}$ be as above, it is straightforward to show $\left(\begin{array}{c}w_{m} \\ \nu_{m \pm} w_{m}\end{array}\right)$ are eigenvectors with eigenvalue $\nu_{m \pm} \cdot$

Definition 3.1. Define $\lambda_{x}: S^{1} \rightarrow \mathbb{C}$ and $\lambda_{v}: S^{1} \rightarrow \mathbb{C}$ by

$$
\lambda_{x}(\phi) \equiv g_{x} \sum_{j \in \mathcal{N}} \rho_{x, j} e^{i j \phi} \text { and } \lambda_{v}(\phi) \equiv g_{v} \sum_{j \in \mathcal{N}} \rho_{v, j} e^{i j \phi},
$$

and define the curve $\gamma \subset \mathbb{C}$ to be the set of all $\nu \in \mathbb{C}$ satisfying

$$
\nu^{2}-\lambda_{v}(\phi) \nu-\lambda_{x}(\phi)=0
$$

for some $\phi \in[0,2 \pi]$. 


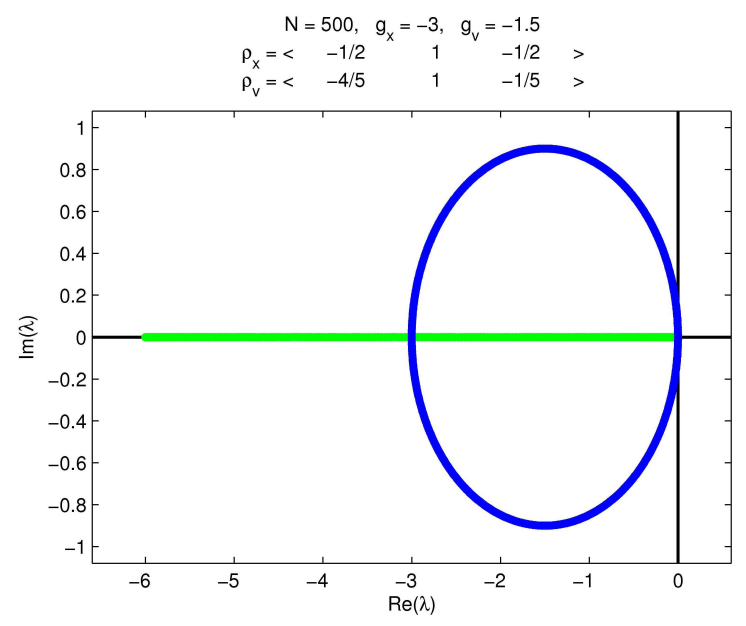

Figure 1: A representative figure for the calculation of the eigenvalues for 500 agents. The values of the parameters are given in the figures. Green line: $\lambda_{x, m}$, Blue ellipse: $\lambda_{v, m}$.

Note first that $\lambda_{x}$ and $\lambda_{v}$ are be independent of $N$ (provided $N$ is larger than half the size of the neighborhood $\mathcal{N}$ ). This implies in turn that $\gamma$ depends on all parameters of the flocking model except $N$. As $N$ grows and the remaining parameters are fixed, the eigenvalues of the associated Laplacians tend to fill out curves denoted by $\lambda_{x}$ and $\lambda_{v}$. This is illustrated in Figure 1, The same holds for the eigenvalues of $M_{N}$ (see Figure 2), in particular :

Corollary 3.3. The set $\operatorname{spec}\left(M_{N}\right)$ of eigenvalues of $M_{N}$ satisfies:

$$
\operatorname{spec}\left(M_{N}\right) \subset \gamma \quad \text { and } \quad \lim _{N \rightarrow \infty} \operatorname{spec}\left(M_{N}\right)=\gamma
$$

where the second limit is taken relative to the Haussforff metric $d_{H}$. Similar results hold for the spectra of $L_{x}$ and $L_{v}$ and the images of the curves $\lambda_{x}$ and $\lambda_{v}$.

Proof. For any $\nu_{m \pm}$, setting $\phi=m \theta$ in Definition 3.1 gives $\lambda_{v}(\phi)=\lambda_{v, m}$ and $\lambda_{x}(\phi)=\lambda_{x, m}$ which shows $\nu_{m \pm} \in \gamma$.

The second assertion is equivalent to showing $\lim _{N \rightarrow \infty} d_{H}\left(\gamma, M_{N}\right)=0$. Let $g$ be the set-valued function from $[0,2 \pi]$ given by $g(\phi)=\left\{\nu: \nu^{2}-\lambda_{v}(\phi) v-\right.$ $\left.\lambda_{x}(\phi)=0\right\}$, so that $\gamma=\cup_{\phi \in[0,2 \pi]} g(\phi)$. As $\lambda_{v}$ and $\lambda_{x}$ are continuous, and roots of polynomials depend continuously on their coefficients, $g$ is continuous. As its domain is compact, it is uniformly continuous. Now fix $\epsilon>0$. For any $\nu \in \gamma, \nu \in g(\phi)$ for some $\phi$, there is $\delta>0$ (independent of $\phi$ ) so that $\left|\phi^{\prime}-\phi\right|<\delta \Longrightarrow d_{H}\left(g\left(\phi^{\prime}\right), g(\phi)\right)<\epsilon$. We may take $N$ large enough so that $\left|\frac{k}{2 \pi N}-\phi\right|<\delta$ for some $k$. As $g\left(\frac{k}{2 \pi N}\right) \subset \operatorname{spec}\left(M_{N}\right)$, this implies there are points in $\operatorname{spec}\left(M_{N}\right)$ that are distance less than $\epsilon$ from $\nu$. As $\nu$ was arbitrary, 


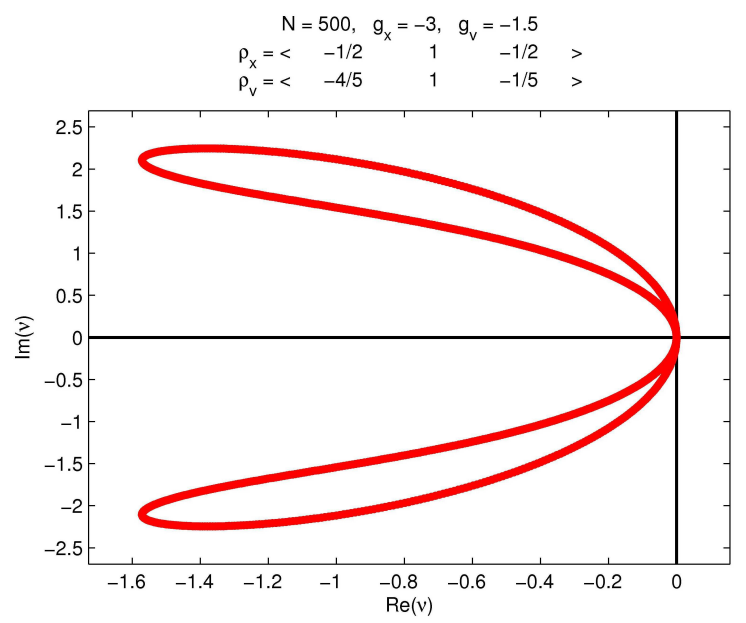

Figure 2: A representative figure for the calculation of the eigenvalues for 500 agents. The values of the parameters are given in the figures. The eigenvalues of $M_{N}$ of Proposition 3.2 .

and we already have $\operatorname{spec}\left(M_{N}\right) \subset \gamma$, this implies $d_{H}\left(\gamma\right.$, spec $\left.\left(M_{N}\right)\right)<\epsilon$, which suffices to prove the desired limit.

We next present a necessary condition for asymptotic stability.

Proposition 3.4. If $I_{x, 1} \neq 0$, then for sufficiently large $N$ the system $S_{N}^{*}$ in Definition 2.1 is not asymptotically stable.

Proof. We will first show that for large enough $N$ and small enough $m$, the eigenvalues $\nu_{m \pm}$ are close to $\pm \sqrt{\lambda_{x}\left(\frac{2 \pi m}{N}\right)}$, which will have positive real part for one branch.

We may write the expansions $\lambda_{x}(\phi)=i \phi I_{x, 1}-\frac{\phi^{2}}{2} I_{x, 2}-\ldots$ and $\lambda_{v}(\phi)=$ $i \phi I_{v, 1}-\frac{\phi^{2}}{2} I_{v, 2}-\ldots$ similar to equation (5). If $I_{x, 1} \neq 0$, these imply existence of constants $A, B$, and $\delta_{1}>0$ so that $|\phi|<\delta_{1}$ implies both $\left|\lambda_{x}(\phi)\right| \geq B|\phi|$ and $\left|\lambda_{v}(\phi)\right| \leq A|\phi|$. Set $\nu_{ \pm}(\phi)=\frac{\lambda_{v}(\phi)}{2} \pm \sqrt{\frac{\lambda_{v}^{2}(\phi)}{4}+\lambda_{x}(\phi)}$ (so that $\nu_{m \pm}=\nu_{ \pm}\left(\frac{2 \pi m}{N}\right)$, as in Proposition 3.2). By taking $|\phi|<\delta_{2}$ we may ensure $\mid \sqrt{\lambda_{x}+\lambda_{v}^{2} / 4}-$ $\sqrt{\lambda_{x}}\left|<\frac{1}{4}\right| \sqrt{\lambda_{x}} \mid$. Then $\left|\nu_{ \pm}(\phi)- \pm \sqrt{\lambda_{x}(\phi)}\right|=\left|\lambda_{v}(\phi) / 2 \pm\left(\sqrt{\lambda_{v}^{2} / 4+\lambda_{x}}-\sqrt{\lambda_{x}}\right)\right| \leq$ $\frac{1}{4}\left|\lambda_{x}\right|+\frac{1}{2} A|\phi|$. As $\left|\sqrt{\lambda_{x}}\right| \geq \sqrt{B|\phi|}$, by taking $|\phi|<\delta_{3}$ we can ensure $\mid \nu_{ \pm}(\phi)-$ $\pm \sqrt{\lambda_{x}(\phi)}\left|\leq \frac{1}{2}\right| \sqrt{\lambda_{x}} \mid$.

For $|\phi|<\delta_{4}$ we can similarly derive the estimate $\left| \pm \sqrt{\lambda_{x}(\phi)}- \pm \sqrt{i \phi I_{x, 1}}\right| \leq$ $\sqrt{i \phi I_{x, 1}} C|\phi|$ for some constant $C$. Now as $\pm \sqrt{i \phi I_{x, 1}}= \pm \sqrt{\phi I_{x, 1}}\left(\frac{\sqrt{2}}{2}+i \frac{\sqrt{2}}{2}\right)$, at least one of these branches must have positive real part. The previous estimates imply that for $|\phi|<\min \left(\delta_{1}, \delta_{2}, \delta_{3}, \delta_{4}\right)$, one of the branches of $\nu_{ \pm}(\phi)$ must have 
positive real part. Thus for sufficiently large $N$, there are eigenvalues of $M_{N}$ with positive real part, so $S_{N}^{*}$ is not asymptotically stable.

In the remainder of this section we use a global method to determine a better condition for asymptotic stability for nearest neighbor systems.

Proposition 3.5. The system $S_{N}^{*}$ of Definition 2.1 is asymptotically stable for all $N$ (all other parameters fixed) if $\rho_{x, j}$ is symmetric, and

$$
\forall \phi \neq 0: \operatorname{Re}\left(\lambda_{x}(\phi)\right)<0 \text { and } \operatorname{Re}\left(\lambda_{v}(\phi)\right)<0 .
$$

Instability will occur for large $N$ if either of the opposite inequalities holds for some $\phi \neq 0$.

Proof. By the Routh-Hurwitz criterion applied to the equation $\nu^{2}-\lambda_{v, m} \nu-$ $\lambda_{x, m}=0$ with complex coefficients (see [5]), we see that all nonzero eigenvalues given in Proposition 3.2 all have negative real parts if and only if for all $m \in$ $\{1, \cdots N-1\}$ we have (in the notation of Equation [5):

$$
\begin{aligned}
\operatorname{Re}\left(\lambda_{v, m}\right) & <0 \\
2 \operatorname{Re}\left(\lambda_{x, m}\right) & <\left|\lambda_{v, m}\right|^{2} \\
\operatorname{Re}\left(\lambda_{x, m}\right) \operatorname{Re}\left(\lambda_{v, m}\right)+\operatorname{Im}\left(\lambda_{x, m}\right) \operatorname{Im}\left(\lambda_{v, m}\right) & >0 \\
\operatorname{Re}\left(\lambda_{x, m}\right)\left[\operatorname{Re}\left(\lambda_{v, m}\right)\right]^{2}+\operatorname{Re}\left(\lambda_{v, m}\right) \operatorname{Im}\left(\lambda_{x, m}\right) \operatorname{Im}\left(\lambda_{v, m}\right)+\left[\operatorname{Im}\left(\lambda_{x, m}\right)\right]^{2} & <0
\end{aligned}
$$

If $\rho_{x, j}$ are symmetric then $\operatorname{Im}\left(\lambda_{x, m}\right)$ is zero for all $m$. In this case the RouthHurwitz conditions reduce to: for all $m \in\{1, \cdots N-1\}, \operatorname{Re}\left(\lambda_{x, m}\right)<0$ and $\operatorname{Re}\left(\lambda_{v, m}\right)<0$. As $\lambda_{x, m}=\lambda_{x}\left(\frac{2 \pi m}{N}\right)$ and $\lambda_{v, m}=\lambda_{v}\left(\frac{2 \pi m}{N}\right)$, stability for all $N$ follows if $\operatorname{Re}\left(\lambda_{x}(\phi)\right)<0$ and $\operatorname{Re}\left(\lambda_{v}(\phi)\right)<0$ for all $\phi \neq 0$ (the case of $\phi=0$ is excluded because the zero eigenvalue is excluded from our definition of asymptotic stability ).

On the other hand, if either $\operatorname{Re}\left(\lambda_{x}(\phi)\right)>0$ or $\operatorname{Re}\left(\lambda_{v}(\phi)\right)>0$ for some $\phi \neq 0$, then as the set of points $\left\{\frac{2 \pi m}{N}\right\}$ for all $N>0$ and $1 \leq m \leq N$ is dense in $[0,2 \pi]$, and the functions $\lambda_{x}$ and $\lambda_{v}$ are continuous, then there must be some $N$ and $m$ so that either $\operatorname{Re}\left(\lambda_{x, m}\right)>0$ or $\operatorname{Re}\left(\lambda_{v, m}\right)>0$, in which case $S_{N}^{*}$ is not asymptotically stable.

We are now in a position to state and prove the main theorem of this section. Recall that we identify $\rho_{x,-1}$ and $\rho_{v,-1}$ with $\rho_{x, N-1}$ and $\rho_{v, N-1}$ in Definition 2.1

Theorem 3.6. Suppose $S_{N}^{*}$ is as defined in Definition [2.1, with $\mathcal{N}=\{-1,0,1\}$. Then $S_{N}^{*}$ is asymptotically stable for all $N$ if and only if $\rho_{x,-1}=\rho_{x, 1}, g_{x} \rho_{x, 0}<0$, and $g_{v} \rho_{v, 0}<0$.

Proof. Let $S_{N}^{*}$ be asymptotically stable for all $N$. First, Proposition 3.4 implies $I_{x, 1}=0$, which for $\mathcal{N}=\{-1,0,1\}$ implies $\rho_{x,-1}=\rho_{x, 1}$. Next, Equation 2 implies that $\rho_{x, 0}=-\left(\rho_{x, 1}+\rho_{x,-1}\right)$, which implies

$$
\begin{aligned}
\operatorname{Re}\left(\lambda_{x}(\phi)\right) & =\operatorname{Re}\left(g_{x}\left(e^{-i \phi} \rho_{x,-1}+\rho_{x, 0}+e^{i \phi} \rho_{x, 1}\right)\right) \\
& =g_{x} \rho_{x, 0}(1-\cos (\phi))
\end{aligned}
$$


Similarly $\operatorname{Re}\left(\lambda_{v}\right)=g_{v} \rho_{v, 0}(1-\cos (\phi))$. As $(1-\cos (\theta))>0$ for $\phi \neq 0$, Proposition 3.5 implies we must have $g_{v} \rho_{v, 0}<0$ and $g_{x} \rho_{x, 0}<0$.

To prove the other direction, let $\rho_{x,-1}=\rho_{x, 1}, g_{x} \rho_{x, 0}<0$ and $g_{v} \rho_{v, 0}<0$. The same calculation as above shows $\operatorname{Re}\left(\lambda_{x}(\phi)\right)<0$ and $\operatorname{Re}\left(\lambda_{v}(\phi)\right)<0$ for $\phi \neq 0$, then Proposition 3.5 implies $S_{N}^{*}$ is asymptotically stable.

\section{Signal Velocities}

The main result of this section is the determination of the signal velocity in asymptotically stable systems as characterized in Theorem 3.6. The signal velocity is the velocity with which disturbances (such as a short pulse) propagate through the flock. In general, signal velocities in dispersive media may be difficult to determine. The reason is that a pulse consists of a superposition of plane waves, typically each with a different phase velocity. If the component plane waves have different phase velocities, the pulse may spread out over time (dispersion), and the determination of arrival time of the signal may becomes problematic. For details we refer to [3].

For nearest neighbor systems of Definition 2.1 we define:

$$
a \equiv \frac{I_{v, 1}^{2}}{4}+\frac{I_{x, 2}}{2}=\frac{\left(\rho_{v, 0}+2 \rho_{v, 1}\right)^{2} g_{v}^{2}}{4}+\frac{-g_{x} \rho_{x, 0}}{2}
$$

Remark: From now on we will restrict our attention to (stable) systems satisfying the conditions of Definition 2.1 and the conclusions of Theorem 3.6. Note that for these systems $a>0$. In order to simplify notation we will also (without loss of generality, because of Theorem (3.6) re-scale $g_{x}$ and $g_{v}$ so that the values of $\rho_{x, 0}$ and $\rho_{v, 0}$ are 1 from now on.

Remark: From the definitions it is clear that $\nu_{N-m, \pm}$ can be identified with $\nu_{-m, \pm}$ and that $\nu_{-m, \pm}$ is the complex conjugate of $\nu_{m \pm}$. It will be convenient in this section to relabel these eigenvalues so that $m$ runs from $\lceil-(N-1) / 2\rceil$ to $\lceil(N-1) / 2\rceil$. For simplicity of notation, we will however write $\sum_{m=\lceil-(N-1) / 2\rceil}^{\lceil(N-1) / 2\rceil}$ as $\sum_{m=-N / 2}^{N / 2}$.

Proposition 4.1. Let $S_{N}^{*}$ as in Definition 2.1 and Theorem 3.6. Then a>0 
and the eigenvalues $\nu_{m \varepsilon}$ of $M_{N}$ can be expanded as (with $\varepsilon= \pm 1$ and $\theta \equiv \frac{2 \pi}{N}$ ):

$$
\begin{aligned}
\nu_{m \varepsilon}=\quad i m \theta & \left(\frac{I_{v, 1}}{2}+\varepsilon a^{1 / 2}\right)+ \\
m^{2} \theta^{2} & \left(-\frac{I_{v, 2}}{4}-\varepsilon \frac{\left(\frac{I_{v, 1} I_{v, 2}}{4}+\frac{I_{x, 3}}{6}\right)}{2 a^{1 / 2}}\right)+ \\
i m^{3} \theta^{3} & \left(-\frac{I_{v, 3}}{12}-\varepsilon \frac{\left(\frac{I_{v, 1} I_{v, 3}}{12}+\frac{I_{x, 4}}{24}+\frac{I_{v, 2}^{2}}{16}\right)}{2 a^{1 / 2}}+\varepsilon \frac{\left(\frac{I_{v, 1} I_{v, 2}}{4}+\frac{I_{x, 3}}{6}\right)^{2}}{8 a^{3 / 2}}\right)+ \\
m^{4} \theta^{4} & \left(\frac{I_{v, 4}}{48}+\varepsilon \frac{\left(\frac{I_{v, 2} I_{v, 3}}{24}+\frac{I_{v, 2} I_{v, 3}}{48}+\frac{I_{x, 5}}{125}\right)}{2 a^{1 / 2}}-\varepsilon \frac{\left(\frac{I_{v, 1} I_{v, 2}}{4}+\frac{I_{x, 3}}{6}\right)\left(\frac{I_{v, 1} I_{v, 3}}{12}+\frac{I_{x, 4}}{24}+\frac{I_{v, 2}^{2}}{16}\right)}{4 a^{3 / 2}}\right. \\
& \left.+\varepsilon \frac{\left.\left(\frac{I_{v, 1} I_{v, 2}}{4}+\frac{I_{x, 3}}{6}\right)^{3}\right)+\cdots}{16 a^{5 / 2}}\right)+\cdots
\end{aligned}
$$

Proof. Expand $\nu_{m \pm}$ given in Proposition 3.2 in powers of $\theta$ using

$$
a \neq 0 \Rightarrow \sqrt{z-a}= \pm i \sqrt{a}\left(1-\frac{z}{2 a}-\frac{z^{2}}{8 a^{2}}-\frac{z^{3}}{16 a^{3}} \cdots\right)
$$

After a substantial but straightforward calculation the result is obtained.

The phase velocity of the time-varying sinusoid $f(x, t)=e^{i(\omega t-\mathrm{k} x)}$ on the real line is defined by the evolution of points of constant phase : $\omega t-\mathrm{k} x(t)=c$, which gives the phase velocity $\omega / \mathrm{k}$.

Disturbances in the positions of agents in the flock may be decomposed in terms of solutions to Equation 1 which are damped sinusoidal waves as functions of time and agent number. We define phase velocity in units of number of agents per unit time, as follows.

Definition 4.1. The set of solutions

$$
z_{k}(t)=A e^{i(\omega t-b k)} e^{-a t}
$$

has phase velocity $\omega / b$.

On our way to studying the propagation velocity of disturbances in the system $S_{N}^{*}$, we will characterize its phase velocities. We first establish the following :

Lemma 4.2. For $S_{N}^{*}$ as in Theorem [3.6, the imaginary parts of the eigenvalues $\nu_{m \pm}$ have opposite signs for $m \neq 0$. 
Proof. Set $\nu_{m+}=\alpha_{1}+i \beta_{1}$ and $\nu_{m-}=\alpha_{2}+i \beta_{2}$. As $\nu_{m \pm}$ are roots of $\nu^{2}-$ $\nu \lambda_{v, m}-\lambda_{x, m}$, and

$\left(\nu-\mu_{1}\right)\left(\nu-\mu_{2}\right)=\nu^{2}-\left(\alpha_{1}+\alpha_{2}+i\left(\beta_{1}+\beta_{2}\right)\right) \nu+\alpha_{1} \alpha_{2}-\beta_{1} \beta_{2}+i\left(\alpha_{1} \beta_{2}+\alpha_{2} \beta_{1}\right)$,

we can identify $\lambda_{x, m}=-\left(\alpha_{1} \alpha_{2}-\beta_{1} \beta_{2}\right)+i\left(\alpha_{1} \beta_{2}+\alpha_{2} \beta_{1}\right)$. We have $\operatorname{Im}\left(\lambda_{x, m}\right)=0$ as $\rho_{x, j}$ is symmetric, so $\alpha_{1} \beta_{2}+\alpha_{2} \beta_{1}=0$. Solving gives $\beta_{1}=-\frac{\alpha_{1}}{\alpha_{2}} \beta_{2}$. But $\alpha_{1}<0$ and $\alpha_{2}<0$ because $S_{N}^{*}$ is asymptotically stable, so $\beta_{1}$ and $\beta_{2}$ have opposite signs.

Lemma 4.3. For $S_{N}^{*}$ as in Theorem [3.6, phase velocities are given by

$$
c_{m+}=\frac{-\operatorname{Im}\left(\nu_{m-}\right)}{m \theta}>0 \quad \text { and } \quad c_{m-}=\frac{-\operatorname{Im}\left(\nu_{m+}\right)}{m \theta}<0
$$

for $1 \leq m \leq \frac{N}{2}$.

Proof. Lemma 4.2 implies $\operatorname{Im}\left(\nu_{m+}\right)$ and $\operatorname{Im}\left(\nu_{m-}\right)$ have opposite signs. Redefine (if necessary, see Proposition 3.2) the subscripts "+" and "-" so that $\nu_{m+}$ has positive imaginary part, and $\nu_{m-}$ has negative imaginary part.

We now derive phase velocities where + denotes going from agent " 0 " towards agent "N". The expression for the $k^{t h}$ entry of the time-evolution of the solution corresponding to the eigenvalue $\nu_{m \pm}$ is (see Proposition 3.1) is (up to an arbitrary multiplicative constant) :

$$
z_{k}=e^{\left(\nu_{m \pm}\right) t} e^{i k m \theta}=e^{\operatorname{Re}\left(\nu_{m \pm}\right) t} e^{i\left(\operatorname{Im}\left(\nu_{m \pm}\right) t+k m \theta\right)}=e^{\operatorname{Re}\left(\nu_{m \pm}\right) t} e^{i\left(\operatorname{Im}\left(\nu_{m \pm}\right) t-(-m \theta) k\right)}
$$

Comparing this to Definition 4.1 shows these two solutions have phase velocities $c_{m,+}$ and $c_{m,-}$ as given in 7 .

From Proposition 4.1 we see that the eigenvalues close to the origin form four branches which intersect at the origin. Namely $\varepsilon$ can be +1 or -1 , and the counter $m$ can be positive or negative. This is illustrated in Figure 2 So for given $|m|$ we get two phase velocities: one in each direction.

Lemma 4.4. For $S_{N}^{*}$ as in Theorem [3.6, the phase velocities $c_{m \varepsilon}$ of Lemma 4.3 can be expanded as $(\varepsilon \in\{-1,1\})$ :

$$
\begin{aligned}
c_{m \varepsilon}= & -\frac{g_{v}\left(1+2 \rho_{v, 1}\right)}{2}+\varepsilon \sqrt{\frac{g_{v}^{2}\left(1+2 \rho_{v, 1}\right)^{2}}{4}-\frac{g_{x}}{2}}+ \\
& m^{2} \theta^{2}\left(\frac{g_{v}\left(1+2 \rho_{v, 1}\right)}{12}-\varepsilon \frac{2 g_{v}^{2}\left(1+2 \rho_{v, 1}\right)^{2}-g_{x}+\frac{3}{2} g_{v}^{2}}{24\left[g_{v}^{2}\left(1+2 \rho_{v, 1}\right)^{2}-2 g_{x}\right]^{1 / 2}}+\varepsilon \frac{g_{v}^{2}\left(1+2 \rho_{v, 1}\right)}{16\left[g_{v}^{2}\left(1+2 \rho_{v, 1}\right)^{2}-2 g_{x}\right]^{3 / 2}}\right) \\
& +\mathcal{O}\left((m \theta)^{4}\right)
\end{aligned}
$$

The real parts of the associated eigenvalues can be expanded as:

$$
\operatorname{Re}\left(\nu_{m \varepsilon}\right)=m^{2} \theta^{2}\left(\frac{g_{v}}{4}+\varepsilon \frac{g_{v}^{2}\left(1+2 \rho_{v, 1}\right)}{4\left[g_{v}^{2}\left(1+2 \rho_{v, 1}\right)^{2}-2 g_{x}\right]^{1 / 2}}\right)+\mathcal{O}\left((m \theta)^{4}\right)
$$


Proof. With the reduction $\rho_{x, 0}=\rho_{v, 0}=1$ as described in the remark in at the beginning of section 4. Theorem 3.6 implies $\rho_{x, 1}=\rho_{x,-1}=-\frac{1}{2}$, and Equation (2) implies $\rho_{v,-1}=-\left(1+\rho_{v, 1}\right)$. We can then compute all of the moments

$$
\begin{array}{ll}
I_{x, j}=(-1)^{j}\left(-\frac{1}{2}\right)+1^{j}\left(-\frac{1}{2}\right) & = \begin{cases}0 & j \text { even } \\
1 & j \text { odd }\end{cases} \\
I_{v, j}=(-1)^{j}\left(-\left(1+\rho_{v, 1}\right)\right)+1^{j} \rho_{v, 1} & = \begin{cases}-1 & j \text { even } \\
1+2 \rho_{v, j} & j \text { odd }\end{cases}
\end{array}
$$

Substituting the expansion from Proposition 4.1 into the expressions for the phase velocity $c_{m \varepsilon}=-\frac{\operatorname{Im}\left(\nu_{m-\epsilon}\right)}{m \theta}$ from Lemma 4.3, and using the above expressions for the moments $I_{x, j}$ and $I_{v, j}$ gives the desired expansion.

For any set of initial conditions $z_{k}(0)$ and $\dot{z}_{k}(0)$, there are unique constants $a_{m}$ and $b_{m}$ so that the solution of the system $S_{N}^{*}$ has the form

$$
z_{k}(t)=\sum_{m=-N / 2}^{N / 2} a_{m} e^{i m \theta k} e^{\nu_{m+} t}+\sum_{m=-N / 2}^{N / 2} b_{m} e^{i m \theta k} e^{\nu_{m-} t}
$$

Our main result of this paper is to show that the first sum represents a signal travelling to the left (decreasing agent number), that may be approximated by a travelling wave with a single signal velocity. Likewise, the second sum represents a signal travelling to the right. We first need a small technical lemma.

Lemma 4.5. There is a $\delta>0$ such that for all a,b satisfying $|a|<\delta$ and $|b|<\delta$, it follows that $\left|e^{a}-e^{b}\right|<2|a-b|$.

Proof. $e^{a}-e^{b}=(a-b)+\sum_{n=2}^{\infty} \frac{1}{n !}\left(a^{n}-b^{n}\right)$. Using $a^{n}-b^{n}=(a-b) \sum_{j=0}^{n-1} a^{j} b^{n-1-j}$ we have $\left|e^{a}-e^{b}\right|=|a-b|\left(1+\sum_{n=2}^{\infty} \frac{1}{n !}\left(\sum_{j=0}^{n-1} a^{j} b^{n-1-j}\right)\right)$. The term multiplying $|a-b|$ expression is a convergent power series, so is continuous, and approaches 1 as $a \rightarrow 0$ and $b \rightarrow 0$. The desired inequality then follows.

We now address the first term in Equation 8 .

Proposition 4.6. Let $S_{N}^{*}$ be as in Theorem 3.6, and $c_{-} \equiv c_{0-}$ as given in Lemma $4.4(m=0)$. Suppose the initial conditions are such that $b_{m}=0$ for all $m$, in the expansion in Equation (8). In addition, suppose that the coefficients $a_{m}$ satisfy $\left|a_{m}\right|<\frac{M}{m^{p}}$ for some $p>1$. Fix $K>1$ and $0<\alpha<\beta<1$. Then, for all $t \in\left[\frac{N}{\left|c_{-}\right|}, K \frac{N}{\left|c_{-}\right|}\right]$, there is a function $f_{-}$so that

$$
\begin{aligned}
\left|z_{k}(t)-f_{-}\left(k-c_{-} t\right)\right|<\frac{M D K}{\left|c_{-}\right|} N^{3 \alpha-1} & +\frac{2 M}{p-1}\left(\left(N^{\alpha}-1\right)^{1-p}-\left(N^{\beta}-1\right)^{1-p}\right) e^{-\mathcal{C}(\alpha, \beta) t} \\
& +\frac{2 M}{p-1}\left(\left(N^{\beta}-1\right)^{1-p}\right) e^{-\mathcal{C}(\beta, 1) t}
\end{aligned}
$$

for sufficiently large $N$, where $D$ is a constant, and

$$
\mathcal{C}(a, b)=\min _{N^{a} \leq|m| \leq \min \left(N / 2, N^{b}\right)}\left|\operatorname{Re}\left(\nu_{m+}\right)\right| .
$$


Proof. Consider the signal after time $t$ :

$$
z_{k}(t)=\left(\sum_{|m|<N^{\alpha}}+\sum_{|m| \in\left[N^{\alpha}, N^{\beta}\right)}+\sum_{|m| \geq N^{\beta}}\right) a_{m} e^{i m \theta k} e^{\nu_{m+} t}
$$

Using Lemma 4.3 we obtain

$$
e^{\nu_{m+} t}=e^{\operatorname{Re}\left(\nu_{m+}\right) t} e^{i \operatorname{Im}\left(\nu_{m+}\right) t}=e^{\operatorname{Re}\left(\nu_{m+}\right) t} e^{-i m \theta c_{m-} t}
$$

Substituting back to Equation (10) gives

$$
z_{k}(t)=\left(\sum_{|m|<N^{\alpha}}+\sum_{|m| \in\left[N^{\alpha}, N^{\beta}\right)}+\sum_{|m| \geq N^{\beta}}\right) a_{m} e^{\operatorname{Re}\left(\nu_{m+}\right) t} e^{i m \theta\left(k-c_{m}-t\right)}
$$

We note that this form shows that the solution is a sum of sinusoids, with phase velocity $c_{m-}$, that are damped by the exponential factor $e^{\operatorname{Re}\left(\nu_{m+}\right)}$. Intuitively, our result follows because for small $m$ the phase velocities are all close to the constant $c_{-}$and the damping is minimal, while for large $m$ the damping is large enough so that we can ignore that the phase velocities depend on $m$.

Set $f_{-}(z)=\sum_{|m|<N^{\alpha}} a_{m} e^{i m \theta z}$. We then see

$$
\begin{aligned}
\left|z_{k}(t)-f_{-}\left(k-c_{-} t\right)\right| \leq \mid & \sum_{|m|<N^{\alpha}} a_{m}\left(e^{\operatorname{Re}\left(\nu_{m+}\right) t} e^{i m \theta\left(k-c_{m}-t\right)}-e^{i m \theta\left(k-c_{-} t\right)}\right) \mid+ \\
& \left|\sum_{|m| \in\left[N^{\alpha}, N^{\beta}\right]} a_{m} e^{\operatorname{Re}\left(\nu_{m}\right) t} e^{i m \theta\left(k-c_{m}-t\right)}\right|+ \\
& \left|\sum_{|m|>N^{\beta}} a_{m} e^{\operatorname{Re}\left(\nu_{m+}\right) t} e^{i m \theta\left(k-c_{m-} t\right)}\right|
\end{aligned}
$$

We will bound these three sums separately. For the first sum, we may factor

$e^{\operatorname{Re}\left(\nu_{m}\right) t} e^{i m \theta\left(k-c_{m-} t\right)}-e^{i m \theta\left(k-c_{-} t\right)}=e^{i m \theta\left(k-c_{m-} t\right)}\left(e^{\operatorname{Re}\left(\nu_{m}\right) t}-e^{i m \theta\left(c_{m-}-c_{-}\right) t}\right)$

Applying lemma 4.5 with $a=\operatorname{Re}\left(\nu_{m+}\right) t$ and $b=i m \theta\left(c_{m-}-c_{-}\right) t$ gives (provided these can be made small enough)

$$
\begin{aligned}
\mid e^{i m \theta\left(k-c_{m-} t\right)}\left(e^{\operatorname{Re}\left(\nu_{m+}\right) t}-e^{i m \theta\left(c_{\left.m_{-}-c_{-}\right) t}\right) \mid}\right. & \leq 2\left|\operatorname{Re}\left(\nu_{m+}\right) t-i m \theta\left(c_{m_{-}}-c_{-}\right) t\right| \\
& \leq 2|t|\left(\left|\operatorname{Re}\left(\nu_{m+}\right)\right|+|m \theta|\left|c_{m-}-c_{-}\right|\right)
\end{aligned}
$$

The expansions in Lemma 4.4 shows that both $\left|c_{m-}-c_{-}\right|$and $\operatorname{Re}\left(\nu_{m+}\right)$ are $\mathcal{O}\left(\theta^{2} m^{2}\right)$. In addition, for $|m|<N^{\alpha},|m \theta|<2 \pi m N^{\alpha-1}$. This implies there is a constant $C$ so that $\left|\operatorname{Re}\left(\nu_{m+}\right)\right|+|m \theta|\left|c_{m-}-c_{-}\right|<C\left(N^{\alpha-1}\right)^{2}$ (and also 
justifies that $a$ and $b$ as defined above may be made sufficiently small, by taking $N$ sufficiently large). The first sum in Equation 12 has $2 N^{\alpha}$ terms, each has $\left|a_{m}\right|<M$; the entire sum is then bounded by $2 N^{\alpha} M 2|t| C\left(N^{\alpha-1}\right)^{2}=$ $4 M|t| C N^{3 \alpha-2}$. For $t \in\left[N /\left|c_{-}\right|, K N /\left|c_{-}\right|\right]$, the same entire sum is bounded by $4 M K\left(N /\left|c_{-}\right|\right) C N^{3 \alpha-2}=\frac{M_{-} D K}{\left|c_{-}\right|} N^{3 \alpha-1}$ for $D=4 C$.

For the second sum, we have $\left|e^{\operatorname{Re}\left(\nu_{m+}\right) t}\right|<e^{-\mathcal{C}(\alpha, \beta) t}$. Using the decay condition on the coefficients $a_{m}$ shows the second sum is bounded by

$$
\left(2 \sum_{m=N^{\alpha}}^{N^{\beta}} M m^{-p}\right) e^{-\mathcal{C}(\alpha, \beta) t}
$$

The elementary bound $\sum_{m=a}^{b} m^{-p} \leq \int_{a-1}^{b-1} x^{-p} d x=\frac{1}{p-1}\left((a-1)^{p-1}-(b-1)^{p-1}\right)$, applied above, gives the stated second term of Equation 9. The third sum is bounded similarly by $\frac{2 M}{p-1}\left(N^{\beta}-1\right)^{1-p} e^{-\mathcal{C}(\beta, 1) t}$, the proposition follows from adding the bounds for all three sums.

We now state the main result of the paper, which shows that in general $z_{k}(t)$ is well approximated by two travelling waves, with two different signal velocities, in opposite directions.

Theorem 4.7. Let $S_{N}^{*}$ be as in Theorem 3.6, $c_{ \pm} \equiv c_{0 \pm}$ as given in Lemma 4.4 Fix $0<\alpha<\beta<1$. Let $\left|a_{m}\right|<M m^{-p}$ and $\left|b_{m}\right|<M m^{-p}$ for $p>1$, where $a_{m}$ and $b_{m}$ are as in Equation 8. Fix $K>1$. Define

$$
\mathcal{C}(a, b)=\min _{N^{a} \leq|m| \leq \min \left(N / 2, N^{b}\right)}\left|\operatorname{Re}\left(\nu_{m \varepsilon}\right)\right|
$$

Then, for sufficiently large $N$, there are functions $f_{-}$and $f_{+}$, and constant $D$ so that

$$
\begin{aligned}
\left|z_{k}(t)-f_{-}\left(k-c_{-} t\right)-f_{+}\left(k-c_{+} t\right)\right| & \leq M D K\left(\frac{1}{\left|c_{-}\right|}+\frac{1}{c_{+}}\right) N^{3 \alpha-1} \\
& +\frac{4 M}{p-1}\left(\left(N^{\beta}-1\right)^{1-p}-\left(N^{\alpha}-1\right)^{1-p}\right) e^{-\mathcal{C}(\alpha, \beta) t} \\
& +\frac{4 M}{p-1}\left(\left(N^{\beta}-1\right)^{1-p}\right) e^{-\mathcal{C}(\beta, 1) t}
\end{aligned}
$$

for all $t \in\left[\frac{N}{\left|c_{-}\right|}, K \frac{N}{\left|c_{-}\right|}\right] \cap\left[\frac{N}{c_{+}}, K \frac{N}{c_{+}}\right]$.

In addition, if $\alpha<1 / 3$, then all terms on the r.h.s. of the above inequality tend to 0 as $N \rightarrow \infty$.

Proof. An analogous result to Proposition 4.6 can be proved for the case when $a_{m}=0$ for all $m$. If we write $z_{k}(t)=z_{k}^{+}(t)+z_{k}^{-}(t)$, where $z_{k}^{-}(t)$ has expansion with all $b_{m}=0$ and $z_{k}^{+}(t)$ has expansion with all $a_{m}=0$, we have

$\left|z_{k}(t)-f_{-}\left(k-c_{-} t\right)-f_{+}\left(k-c_{+} t\right)\right| \leq\left|z_{k}^{+}(t)-f_{+}\left(k-c_{+} t\right)\right|+\left|z_{k}^{-}(t)-f_{+}\left(k-c_{-} t\right)\right|$.

Using Proposition 4.6 and the aforementioned analogous result to bound the two terms on the right establishes Equation 13. 
If $\alpha<1 / 3$, then $N^{3 \alpha-1} \rightarrow 0$ as $N \rightarrow \infty$. For $p>1$, both $\left(N^{\beta}-1\right)^{1-p} \rightarrow 0$ and $\left(N^{\alpha}-1\right)^{1-p} \rightarrow 0$, as $N \rightarrow \infty$, which proves that all terms on the r.h.s of Equation 13 go to zero as $N \rightarrow \infty$.

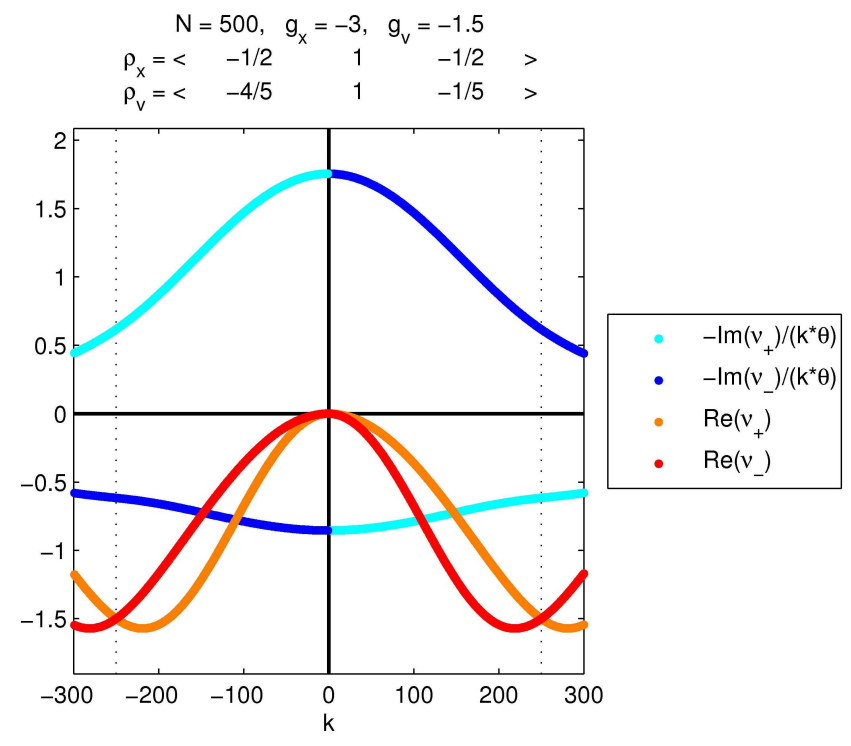

Figure 3: (color online) A representative figure for the calculation of the phase velocities for 500 agents. The values of the parameters are given in the figures. Light blue: $\frac{-\operatorname{Im}\left(\nu_{m+}\right)}{m \theta}$, Blue: $\frac{-\operatorname{Im}\left(\nu_{m-}\right)}{m \theta}$, Orange: $\operatorname{Re}\left(\nu_{m+}\right), \operatorname{Red}: \operatorname{Re}\left(\nu_{m-}\right)$. The maximum phase velocities occur at $m=0$, These are the signal velocities $c_{+}>0$ and $c_{-}<0$ of Theorem 4.7.

Remark: It is interesting to note that the signal velocity we determine is actually equal to the group velocity at $m=0$. The group velocity is defined as $\frac{d c_{m \pm}}{d(m \theta)}$. It is not necessarily true that group velocity in these kinds of systems equals signal velocity. In the system studied in [19] they are different. See [3] for more information.

Remark: A similar argument as the one in Theorem 4.7 easily shows that eigenfunctions with wave numbers $m$ greater than $N^{0.5+\delta}$ will die out before $t=N / c_{+}$. Thus for considerations on time-scales longer than that, these are irrelevant. It also (conveniently) turns out that very often the greatest phase velocities are associated with the lowest wave numbers. A typical case is seen in Figure 3. One can show that in those asymptotically stable cases where $\rho_{v, 1}$ is close to $-1 / 2$, we have that $c_{m \pm}$ has a local maximum at $m=0$. In fact Lemma 
4.4 implies that for $\rho_{v, 1}=-1 / 2$ :

$$
c_{m \varepsilon}=\varepsilon \sqrt{\frac{-g_{x}}{2}}+\varepsilon m^{2} \theta^{2}\left(\frac{g_{x}-\frac{3}{2} g_{v}^{2}}{24 \sqrt{-2 g_{x}}}\right)+\cdots
$$

which has a local maximum at $m=0$.

\section{Conclusion}

Though experiments with cars have been done on circular roads (see [14]), our interest in the system with periodic boundary conditions of $S_{N}^{*}$ as defined in Definition 2.1 stems from the applicability to traffic systems with non-periodic boundary conditions. The primary motivation for studying the former is that they enable us to analyze how disturbances propagate, and - under the assumption that this propagation does not depend on boundary conditions - apply that to the latter systems to find the transients. Some remarks on how that works are given in the Introduction and is the subject of [4]. A relative novelty here is that we consider all strictly decentralized systems, not just symmetric ones.

In Section 3 we give precise conditions on the parameters so that decentralized systems with periodic boundary condition are asymptotically stable. In its generality stated here this is new, though related observations have been made in 2 and 11. The main importance here is that we use these conditions on the parameters to show that in these systems disturbances travel with constant a constant signal velocity, and - as our main result — we determine that velocity in Section 4. This explains why in these cases, approximations of these systems with large $N$, by the wave equation are successful (see for example [2]). It can be shown however that for other parameter values diffusive behavior may occur (see 4 .

Finally we test our prediction of the signal velocity in a numerical experiment. Our theory described the error due to approximating the disturbance signal as having a pair of signal velocities $c_{ \pm}$as a sum of three terms (see equation 13), which asymptotically go to zero for large $N$, subject to a constraint on the decay of the Fourier coefficients of the initial disturbance. In this numerical experiment we give agent number $0=N$ at time $t=0$ is a different initial velocity from the others. We note that even though this type of impulse disturbance does not have the Fourier coefficient decay required by our theory, we nonetheless observe two distinct signal velocities as predicted. The result can be seen in Figure 4. That signal propagates forward (in the direction $1,2,3, .$. ) through the flock as well as backwards (in the direction $N-1, N-2, N-3, \ldots$ ). In figure we color coded according to the speed of the agents, who are stationary until the signal reaches them. In black we mark when the signal is predicted to arrive, according to the theoretically predicted signal velocities. One can see the excellent agreement. 


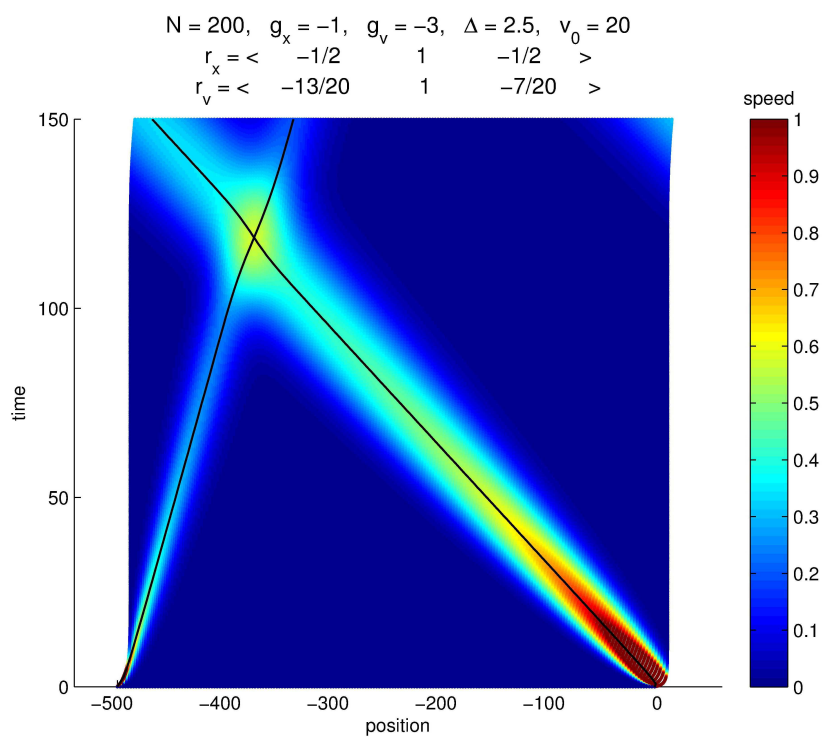

Figure 4: (color online) The orbits of 200 cars with specific choices for the parameters. ( $\Delta$ is the desired distance between cars.) At time 0 agent 0 receives a different initial condition. They are color coded according to the velocity of the agent. The black curves indicate the theoretical position of the wavefront calculated via the signal velocity. Note that these velocities depend on the direction, and that the signal velocity is measured in number of cars per time unit. Due to the different velocities of the cars, these curves are not straight lines.

\section{References}

[1] B. Bamieh, M. R. Jovanović, P. Mitra, S. Patterson, Coherence in LargeScale Networks: Dimension Dependent Limitations of Local Feedback, IEEE Trans. Autom. Contr. 57, Vol 9, 2235-2249, 2012.

[2] P. Barooah, P. G. Mehta, J. P. Hespanha, Mistuning-Based Control Design to Improve Closed-Loop Stability of Vehicular Platoons, IEEE Trans. Autom. Contr. 54, 2100-2113, 2009.

[3] L. Brillouin, Propagation of Electro-Magnetic Waves in Material Media, Congrès International d'Electricité Vol 2, 739-788, 1933. Also appeared in: L. Brillouin, Wave Propagation and Group Velocity, Academic Press, 1960 .

[4] C. E. Cantos, J. J. P. Veerman, Transients in the Synchronization of Oscillator Arrays, Submitted. 
[5] G. Lafferriere, A. Williams, J. S. Caughman, J. J. P. Veerman, Decentralized Control of Vehicle Formations, Systems \& Control Letters, 54, Issue 9, 899-910, 2005.

[6] R. E. Chandler, R. Herman, E. W. Montroll, Traffic Dynamics: Studies in Car Following, Operations Research 6, 165-184, 1958.

[7] K-C. Chu, Decentralized Control of High-Speed Vehicle Strings, Transportation Science 8, No 4, 361-384, 1974.

[8] P. A. Cook, Conditions for String Stability, Syst. Contr. Lett. 54, 991998, 2005.

[9] R. Herman, E. W. Montroll, R. B. Potts, R. W. Rothery, Traffic Dynamics: Analysis and Stability in Car Following, Operations Research 7, 86-106, 1959.

[10] I. Kra, S. R. Simanca, On Circulant Matrices, Notices AMS, March 2012, 368-377.

[11] F. Lin, M. Fardad, M. R. Jovanović, Optimal Control of Vehicular Formations with Nearest Neighbor Interactions, IEEE Trans. Autom. Contr. 57, No 9, 2203-2218, 2012.

[12] N. Mitarai, H. Nakanishi, Convective Instability and Structure Formation in Traffic Flow, J. Phys. Soc. of Japan 69, No 11, 3753-3761, 2000.

[13] C. Robinson, Dynamical Systems, Stability, Symbolic Dynamics, and Chaos, 2nd Edn, CRC Press, 1999.

[14] Y. Sugiyama, M. Fukui, M. Kikuchi, K. Hasebe, A. Nakayama, K. Nishionari, S. Tadaki, S. Yukawa, Traffic Jams without Bottlenecks - Experimental Evidence for the Physical Mechanism of the Formation of a Jam, New Journal of Physics 10, No 3, 033001, 2008.

[15] F. M. Tangerman, J. J. P. Veerman, B. D. Stočić, Asymmetric Decentralized Flocks, Trans. Autom. Contr. 57, No 11, 2844-2854, 2012.

[16] L. N. Trefethen, Pseudospectra of Linear Operators, SIAM Review 39, No 3, pp. 383-406, September 1997.

[17] J. J. P. Veerman, J. S. Caughman, G. Lafferriere, A. Williams, Flocks and Formations, J. Stat. Phys. 121, Vol 5-6, 901-936, 2005.

[18] J. J. P. Veerman, Symmetry and Stability of Homogeneous Flocks (a Position Paper), Proc. 1st Int'l Conf. on Pervasive and Embedded Computing and Communication Systems, Algarve, 2010.

[19] J. A. Ward, R. E. Wilson, Criteria for Convective versus Absolute String Instability in Car-Following Models, Proc. Royal Soc. A 467, 2185-2208, 2011. 